\title{
Dual-Targeted Molecular Imaging of Cancer
}

\author{
Emily B. Ehlerding ${ }^{1}$, Lingyi Sun $^{2}$, Xiaoli Lan ${ }^{3}$, Dexing Zeng ${ }^{2}$, and Weibo Cai ${ }^{1,4,5}$ \\ ${ }^{1}$ Department of Medical Physics, University of Wisconsin-Madison, Madison, Wisconsin; ${ }^{2}$ Department of Medicine, University \\ of Pittsburgh, Pittsburgh, Pennsylvania; ${ }^{3}$ Department of Nuclear Medicine, Union Hospital, Tongji Medical College, Huazhong \\ University of Science and Technology, Wuhan, China; ${ }^{4}$ Department of Radiology, University of Wisconsin-Madison, Madison, \\ Wisconsin; and ${ }^{5}$ Carbone Cancer Center, University of Wisconsin-Madison, Madison, Wisconsin
}

\begin{abstract}
Molecular imaging is critical to personalized and precision medicine. Although singly targeted imaging probes are making an impact both clinically and preclinically, molecular imaging strategies using bispecific probes have enabled improved visualization of cancer in recent years through synergistic targeting of two ligands. In this Focus on Molecular Imaging review, we outline how peptide-, antibody-, and nanoparticle-based platforms have affected this emerging strategy, providing examples and pointing out areas in which the greatest clinical impact may be realized.
\end{abstract}

Key Words: molecular imaging; dual-targeted; bispecific antibody fragment; cancer; positron emission tomography

J Nucl Med 2018; 59:390-395

DOI: 10.2967/jnumed.117.199877

A

s personalized and precision medicine have come to the forefront of cancer therapies, a need for targeted imaging agents has also arisen. Great advances have been made in molecular imaging using highly specific peptide-, antibody-, and nanoparticle-based agents, enabling noninvasive, longitudinal, in-depth information in preclinical cancer models, as well as clinically about a patient's disease state. In some instances, however, greater imaging contrast and higher specificity for diseased tissues are still warranted. Dual-targeted molecular imaging agents have filled this vacancy, providing specific uptake in tissues of interest that is superior to their corresponding monomeric counterparts due to their increased maximum binding and improved pharmacokinetic profiles. While the affinity of each monomer is ideally maintained, specific interaction with two different targets allows these dual-targeted probes to display enhanced avidity. These agents may target two receptors/proteins on a cancer cell's surface, the tumor microenvironment, or on immune cells.

\footnotetext{
Received Oct. 13, 2017; revision accepted Dec. 17, 2017.

For correspondence or reprints contact either of the following: Dexing Zeng, Department of Medicine, University of Pittsburgh, BST3, \#10015, 3501 Fifth Ave., Pittsburgh, PA 15260.

Email: zengd@upmc.edu

Weibo Cai, Department of Radiology and Medical Physics, University of Wisconsin-Madison, 1111 Highland Ave., Madison, WI 53705.

Email:WCai@uwhealth.org

Published online Jan. 4, 2018.

COPYRIGHT (c) 2018 by the Society of Nuclear Medicine and Molecular Imaging.
}

In addition to molecular imaging, dual-targeting strategies have also been applied to generate therapeutic agents for disease treatment. More than 60 bispecific antibodies have been developed for potential therapeutic purposes (1). One of the major applications of bispecific antibodies is for redirecting immune effector cells to tumor cells. Bispecific T-cell engagers, binding to $\mathrm{T}$ cells (e.g., via the CD3 receptor) and tumor cells (via a tumor-specific target), direct $\mathrm{T}$ cells to tumor cells for cytotoxic activity; among them, blinatumomab was approved by the Food and Drug Administration in 2014 for acute B-cell lymphoblastic leukemia treatment (2). An additional therapeutic application of bispecific antibodies is for simultaneously blocking two different signaling pathways, a feat that could not be achieved with a monospecific antibody. One representative of this type of antibody is duligotuzumab, which targets both the epidermal growth factor receptor (EGFR) and the human epidermal growth factor receptor 3 (HER3), overcoming the drug resistance often encountered by patients receiving the anti-EGFR drug cetuximab (3).

Of note, bispecific platforms are being used in a unique manner for pretargeting strategies (4). Therein, an agent that is targeted to a cancer cell surface marker and also has specificity for a biorthogonal compound (such as a chelator) is injected and allowed to accumulate at a tumor site and clear elsewhere. After this, the second compound, with an imaging tag attached (such as a PET isotope), is injected and preferentially accumulates at the tumor site. Promising preclinical and initial clinical results have been reported, making this another area for bispecific tracers to make an impact in future cancer care.

Collectively, dual-targeted strategies have been demonstrated to be promising approaches for both imaging and therapy. Therapeutic applications of bispecific agents have been thoroughly outlined elsewhere (5); therefore, we herein aim to provide a brief overview of the uses and development of dual-targeted molecular imaging agents, based on peptide, antibody, and nanoparticle platforms (Table 1).

\section{PEPTIDE-BASED AGENTS}

Peptide-based heterodimeric tracers have played an important role in dual-targeted molecular imaging. Although reports have been made with other modalities $(6)$, herein we focus on PET imaging with these heterodimers, as they may 
TABLE 1

Representative Examples of Dual-Targeted Molecular Imaging Agents

\begin{tabular}{|c|c|c|}
\hline Agent & Imaging modality & Reference \\
\hline \multicolumn{3}{|l|}{ Peptide-based } \\
\hline \multicolumn{3}{|l|}{ Targets } \\
\hline PSMA and GRPR & PET & 7 \\
\hline $\begin{array}{l}\text { GRPR and integrin } \\
\qquad \alpha_{v} \beta_{3}\end{array}$ & PET & $11-13$ \\
\hline $\begin{array}{l}\text { uPAR and integrin } \\
\alpha_{v} \beta_{3}\end{array}$ & PET & 8 \\
\hline \multicolumn{3}{|l|}{$\begin{array}{l}\text { Antibody- and antibody } \\
\text { fragment-based }\end{array}$} \\
\hline \multicolumn{3}{|l|}{ Targets } \\
\hline EGFR and HER2 & $\begin{array}{l}\text { PET/SPECT, } \\
\text { fluorescence }\end{array}$ & $16-18$ \\
\hline HER2 and HER3 & SPECT & 19 \\
\hline TF and CD105 & PET/fluorescence & 20 \\
\hline EGFR and CD105 & PET/fluorescence & 21 \\
\hline $\begin{array}{l}\text { EGFR and integrin } \\
\alpha_{v} \beta_{3} / \beta_{5}\end{array}$ & MRI & 22 \\
\hline $\mathrm{CD} 3 \varepsilon$ and $\mathrm{CEA}$ & Fluorescence & 23 \\
\hline $\mathrm{CD} 3 \varepsilon$ and $\mathrm{EpCAM}$ & PET/fluorescence & 24 \\
\hline $\mathrm{CD} 3 \varepsilon$ and NY-ESO-1 & Fluorescence & 25 \\
\hline \multicolumn{3}{|l|}{ Nanoparticle-based } \\
\hline \multicolumn{3}{|l|}{ Targets } \\
\hline $\begin{array}{l}\text { CD44 and integrin } \\
\alpha_{v} \beta_{3}\end{array}$ & Fluorescence & 27,28 \\
\hline FR and integrin $\alpha_{v} \beta_{3}$ & Fluorescence & 29 \\
\hline FR and CD44 & Fluorescence & 30 \\
\hline CD44 and GAR & Fluorescence & 33 \\
\hline FR and integrin $\alpha_{v} \beta_{3}$ & $\begin{array}{l}\text { Fluorescence } \\
\text { and US }\end{array}$ & 34 \\
\hline $\begin{array}{l}\text { Neurokinin-1 and } \\
\text { integrin } \alpha_{v} \beta_{3}\end{array}$ & SPECT and MRI & 35 \\
\hline $\begin{array}{l}\text { P-selectin and integrin } \\
\alpha_{v} \beta_{3}\end{array}$ & Fluorescence & 36 \\
\hline
\end{tabular}

$\mathrm{TF}=$ tissue factor; CEA = carcinoembryonic antigen; US = ultrasound; $F R=$ folate receptor.

be most readily translated to the clinic. The major synthetic strategies for these peptide-based agents can be categorized as using either bifunctional chelators (BFCs) or amino acids as frameworks. The major advantage of using a BFC is that the BFC itself serves as both the linker and the chelator, simplifying the synthetic procedure (Fig. 1A). Eder's group reported $N, N^{\prime}$-bis(2-hydroxy-5-(carboxyethyl)benzyl)ethylenediamine- $N, N^{\prime}$-diacetic acid ([HBED-CC] TFP $_{2}$ ) as an ideal $\mathrm{BFC}$ for constructing peptide-based heterodimeric probes, as it bears two leaving groups for nucleophilic attack by primary amines at both sides of the chelator (7). Another NOTA-based BFC, 4-azido-2-(4,7-bis(2-(tert-butoxy)2-oxoethyl)-1,4,7-triazonan-1-yl)butanoic acid ([ $\left.\left.\mathrm{N}_{3}-\mathrm{NO}^{\mathrm{t}} \mathrm{B}_{2}\right]\right)$, was developed by Zeng's group (8). Unlike (HBED-CC) $\mathrm{TFP}_{2}$, which possesses two identical reactive groups, $\mathrm{N}_{3^{-}}$ $\mathrm{NO}^{t} \mathrm{~B}_{2}$ contains two different reactive groups including an $\mathrm{N}_{3}$ group for click chemistry and a $\mathrm{COOH}$ group for amide coupling, offering selectivity for conjugation with two different peptides. In addition to BFCs, amino acids such as lysine (9) and glutamate (10) have been developed as frameworks, providing flexibility to select an appropriate chelator based on the radioisotope to fulfill different experimental requirements.

One example of a peptide-based PET tracer is the RGD$\mathrm{BBN}(\mathrm{RGD}=$ Arg-Gly-Asp; $\mathrm{BBN}=$ bombesin) heterodimer that was initially developed by Li et al. Using the glutamatebased framework, the resulting dimeric RGD-BBN could simultaneously bind both gastrin-releasing peptide receptor (GRPR) and integrin $\alpha_{v} \beta_{3}$ (11). Both ${ }^{18} \mathrm{~F}-(12)$ and ${ }^{64} \mathrm{Cu}-$ (11) labeled RGD-BBN heterodimers were successfully prepared and evaluated in PC-3 xenograft mouse models. Very recently, the dimeric RGD-BBN probe was ${ }^{68} \mathrm{Ga}-$ labeled, and the resulting PET tracer has been successfully used for clinical PET imaging of prostate cancer patients (13), showing clear uptake in tumors with an $\mathrm{SUV}_{\max }$ near 4.5 for primary tumors and 6.3 for metastatic lymph nodes (Fig. 1B).

Another recent example is the RGD-AE105 heterodimer that was prepared by Gai et al., using the BFC framework $\mathrm{N}_{3}-\mathrm{NO}^{t} \mathrm{~B}_{2}(8)$. The prepared RGD-AE105 tracer targets both the integrin $\alpha_{v} \beta_{3}$ and the urokinase-type plasminogen activator receptor simultaneously. Saturation binding assay results revealed a smaller equilibrium dissociation constant and larger receptor density for the RGD-AE105 heterodimer than for the two corresponding monomers, indicating the increased binding avidity achieved by the RGD-AE105 heterodimer. The in vivo imaging results, obtained from mice bearing U87MG xenografts (overexpressing both targets), showed the superior tumor uptake of the heterodimer (3.27 percentage injected dose per gram [\% ID/g]) compared with that of corresponding RGD or AE105 monomers (1.55 $\% \mathrm{ID} / \mathrm{g}$ for RGD and $1.73 \% \mathrm{ID} / \mathrm{g}$ for AE105), demonstrating the potential for integrin $\alpha_{\mathrm{v}} \beta_{3}$ and urokinase plasminogen activator receptor dual-targeted PET imaging (Fig. 1C). The other two reported RGD-containing bispecific tracers are RGD-octreotate, targeting the somatostatin receptor subtype 2 (9), and RGD-GE11, targeting EGFR (14). The frequent use of RGD for preparing heterodimers suggests its excellent tumor-targeting properties and good pharmacokinetic profile.

Other bispecific agents not containing the RGD motif have also been reported. One representative example is a heterodimer developed by Kopka's group, targeting both prostate-specific membrane antigen (PSMA) and GRPR (7). Specifically, a urea-based PSMA inhibitor was conjugated with the nonapeptide BZH3 targeting GRPR, using (HBED$\mathrm{CC}) \mathrm{TFP}_{2}$ to generate this heterodimer. Further in vivo evaluation revealed its promising uptake in both PSMA-positive and GRPR-positive tumors $(5.4 \% \mathrm{ID} / \mathrm{g}$ for PSMA-positive and $3.3 \% \mathrm{ID} / \mathrm{g}$ for GRPR-positive tumors), which suggests application in prostate cancer detection, since both PSMA and 


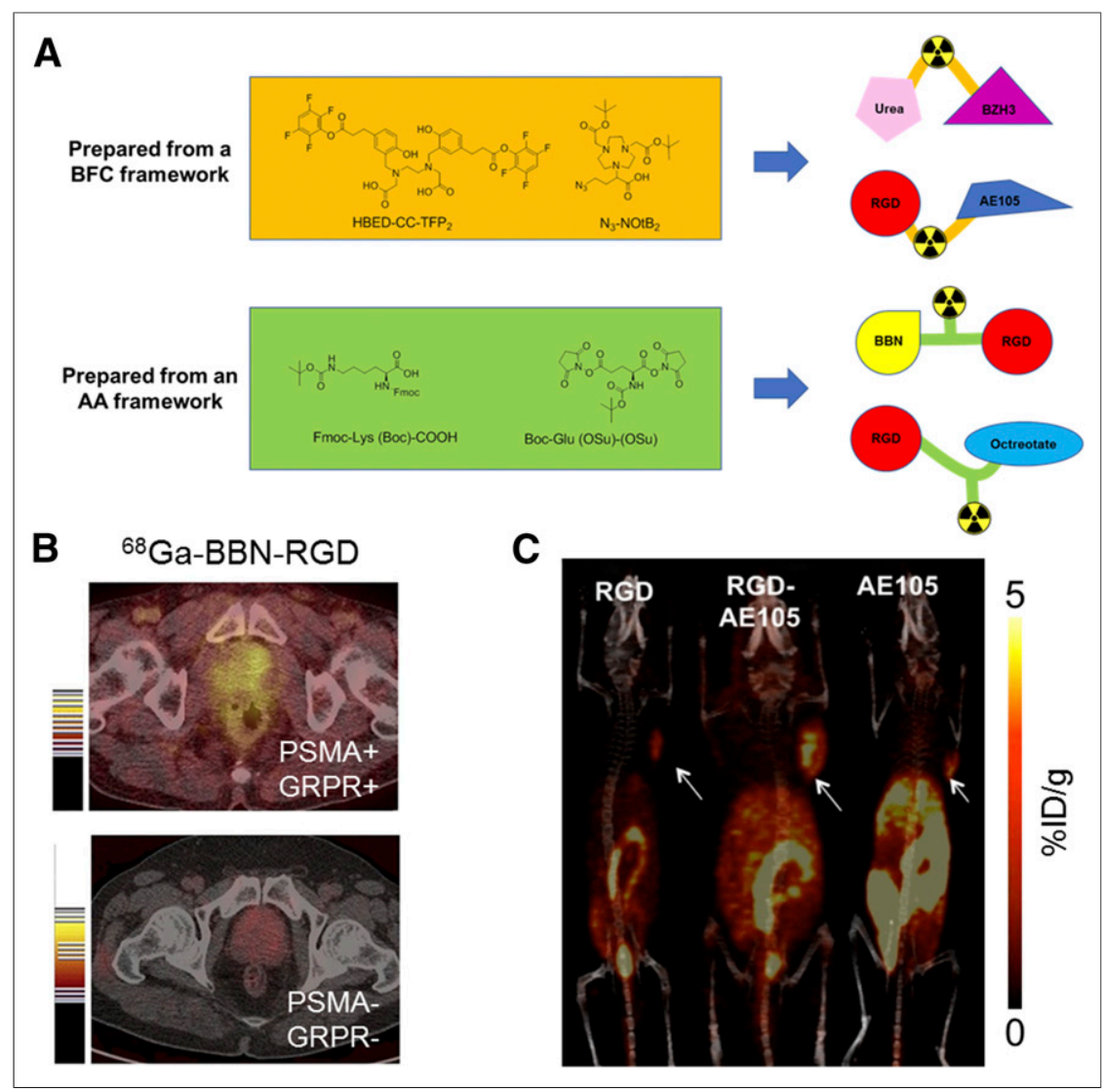

FIGURE 1. (A) Bifunctional chelator and amino acid frameworks for preparing peptide-based dual-targeted imaging agents. (B) PET imaging of ${ }^{8} \mathrm{Ga}-\mathrm{BBN}-\mathrm{RGD}$ in a GRPR- and integrin $\alpha_{v} \beta_{3}$-positive clinical prostate cancer patient (top) and doubly negative patient (bottom) (13). (C) PET images of U87MG-bearing mice (arrows) at $4 \mathrm{~h}$ after injection of indicated ${ }^{64} \mathrm{Cu}$-labeled tracers (8).

GRPR were found to be expressed at a high level in prostate cancer cells.

\section{ANTIBODY- AND ANTIBODY FRAGMENT-BASED AGENTS}

With their excellent target specificity, antibody- and antibody fragment-based bispecific molecular imaging agents have gained popularity in the last decade. The synthesis mechanisms for these tracers have been thoroughly outlined elsewhere (15). In general, these agents may fall into two classes: those that target two antigens on cancer cells, and those that target a cancer cell and another type as well. The advantages of dually targeting cancer cells are clear, enabling enhanced specificity and higher image quality. This strategy has been preclinically applied to radionuclidebased imaging, fluorescence imaging, and MRI.

HER2 has been identified as an important therapeutic target in breast cancer and, as such, is also a target of several bispecific imaging agents. Reilly's group combined a Fab fragment of trastuzumab (targeting HER2) with epidermal growth factor (EGF, targeting its receptor EGFR) through a PEG linker and labeled with ${ }^{64} \mathrm{Cu}$ for PET imaging (16) and an ${ }^{111}$ In-labeled version for SPECT (17). In
EGFR/HER2-positive tumors with the ${ }^{64} \mathrm{Cu}$-labeled version, the bispecific tracer showed uptake of $4.9 \% \mathrm{ID} / \mathrm{g}$, compared with $1.9 \% \mathrm{ID} / \mathrm{g}$ for only HER2 targeting, or $0.7 \% \mathrm{ID} / \mathrm{g}$ for the EGF monomer. The inclusion of a long PEG linker in the heterodimer was found to prolong blood circulation time and accordingly increase tumor uptake. Similarly, a multivalent antibody was developed by Ding et al. to target HER2 and EGFR through the conjugation of an anti-EGFR Nanobody to two anti-HER2 Affibody molecules, and imaged through an attached fluorescent dye (Fig. 2A) (18). A SPECT agent was also developed to target both HER2 and HER3 for enhanced breast cancer imaging (19).

Other bispecific antibody agents have been fabricated that target not only cancer cells, but also their associated angiogenic vessels. CD105, expressed on neovasculature, has been one of the targets of bispecific agents for PET and fluorescence dual-modality imaging by our group (20,21). Pancreatic cancer models were successfully imaged using a CD105- and tissue factor-targeted bispecific antibody fragment (20), whereas brain cancer was imaged through targeting of CD105 and EGFR with a tracer generated through click chemistry techniques (Fig. 2B) (21). The combination of CD105 and EGFR targeting allowed peak tracer uptake of over $47 \% \mathrm{ID} / \mathrm{g}$ at 15 $\mathrm{h}$ after injection in subcutaneous U87MG tumors, whereas single targeting to either EGFR or CD105 provided significantly lower uptake, demonstrating the synergy that can be achieved through dual targeting. Integrin $\alpha_{v} \beta_{3}$ has also been used for targeting of bispecific imaging agents in combination with EGFR in MRI studies (Fig. 2C) (22).

Antibodies targeting both a cancer cell and an immune cell have shown immense promise in therapeutic settings. The therapeutic mechanisms and effects of bispecific T-cell constructs have been elucidated through direct imaging with the agents preclinically. This has been explored using antibodies targeting $\mathrm{CD} 3 \varepsilon$ on $\mathrm{T}$ cells along with carcinoembryonic antigen in fluorescence imaging (23), EpCAM for both PET and fluorescence imaging (Fig. 2D) (24), and a cancer testis antigen, NY-ESO-1, for fluorescence imaging (25).

\section{DUAL-TARGETED NANOPARTICLES}

Nanoparticles have demonstrated their potential as molecular imaging contrast agents in cancer in nearly every medical imaging modality. Just as with other dual-targeted 


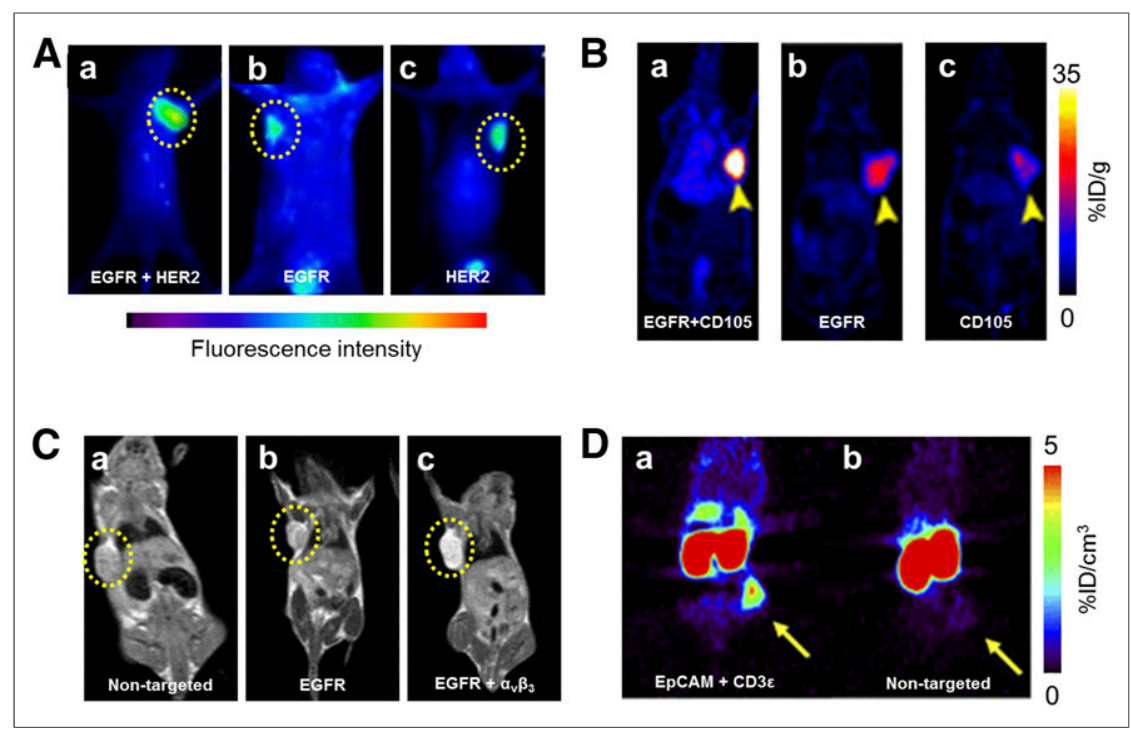

FIGURE 2. (A) Fluorescence imaging of $A 549$ tumors at $8 \mathrm{~h}$ after injection of a tracer for EGFR and HER2 (a), EGFR (b), and HER2 (c) (18). (B) PET imaging of U87MG tumors at $36 \mathrm{~h}$ after injection shows differences in uptake of agent targeting EGFR and CD105 (a), EGFR (b), and CD105 (c) (21). (C) $\mathrm{T}_{1}$ MR images of BGC-823 tumors $1 \mathrm{~h}$ after injection of tracers specific for no targets (a), EGFR alone (b), or both EGFR and $\alpha_{v} \beta_{3}$ (c) (22). (D) PET images $24 \mathrm{~h}$ after injection of bispecific EpCAM/CD3 $\varepsilon$ antibody (a) or isotype control (b) in HT-29 tumor-bearing mice (24).

platforms, the use of two targeting moieties enables nanoparticles to accumulate at a higher level in regions expressing both targets. Nanoparticles are uniquely well-suited for advanced surface modifications, such as conjugation of targeting ligands. This makes dual-targeting with these platforms straightforward in some situations (26). Depending on the nanoparticle and its surface properties, biomolecules may be attached using several strategies, via covalent or noncovalent techniques.

Most reported probes provide contrast in fluorescence imaging, with a few reports in other realms. Similarly, the choices of targeting ligands for dual-targeted nanoparticles have been rather limited. Peptides have been the ligand of choice due to their specificity and small size. Specifically, the targets integrin $\alpha_{v} \beta_{3}, C D 44$, and the folate receptor (FR) have been most thoroughly explored.

For example, CD44 and integrin $\alpha_{v} \beta_{3}$ have been the targets of two nanoparticle platforms: a core-shell system for gene delivery in melanoma models (27) and solid lipid nanoparticles for delivery of docetaxel (28). Self-assembled hydrogel nanoparticles were dually targeted to integrin $\alpha_{\mathrm{v}} \beta_{3}$ and FR, loaded with paclitaxel, and validated in preclinical hepatocellular carcinoma models by Shu et al. (Fig. 3A) (29). Ceramide nanoparticles were developed by Lee et al. and targeted to both CD44 and FR in ovarian cancer (30), and persistent luminescent nanoparticles were targeted to FR and CD44 by Zhao et al. (31). In all these studies, enhanced accumulation in cancerous tissues was demonstrated for dual-targeted nanoparticles over their single or nontargeted counterparts, as evidenced through fluorescence imaging. Other nanoparticles targeting combinations of integrin $\alpha_{v} \beta_{3}$ and EGFR (32) and CD44 along with glycyrrhetinic acid receptors (Fig. 3B) (33) have been imaged using fluorescence as well.

Other imaging modalities have also been used to evaluate dual peptideconjugated nanoparticles. Nanoparticles that were both ultrasound and fluorescence-active were targeted to FR and integrin $\alpha_{v} \beta_{3}$ (Fig. 3C) (34), whereas peptides targeting integrin $\alpha_{\mathrm{v}} \beta_{3}$ and neurokinin-1 receptors allowed loaded liposomes to be visualized by SPECT/ CT and MRI (35). Similarly, liposomes targeting P-selectin and integrin $\alpha_{\mathrm{v}} \beta_{3}$ enabled imaging of metastatic breast cancer models through fluorescence imaging and $\gamma$-scintigraphy (36).

\section{CONCLUSIONS AND FUTURE DIRECTIONS}

Dual-targeted imaging tracers hold immense potential to increase image quality in various diseases, especially in cancer. Although patients may be grouped into a single category based on disease site, it is becoming increasingly obvious that the heterogeneity of cancer will need to be addressed for successful therapy and personalized/precision medicine. Within a single anatomic site, a particular patient,

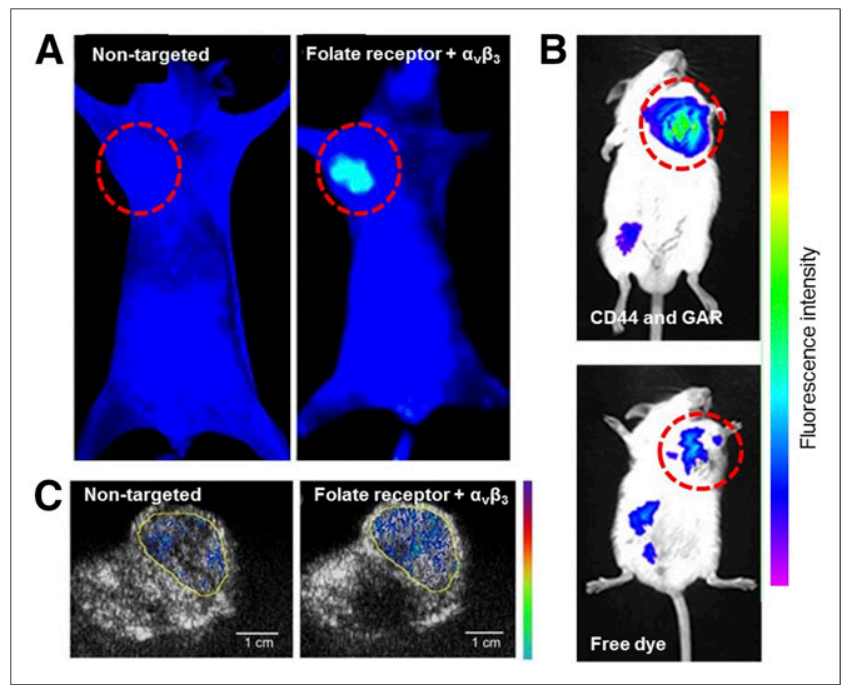

FIGURE 3. Dual-targeted nanoparticle imaging agents. (A) Fluorescence imaging of tumor-bearing mice $48 \mathrm{~h}$ after injection of nontargeted hydrogels (left) or FR and $\alpha_{v} \beta_{3}$-targeted nanoparticles (right) (29). (B) Fluorescence imaging of $\mathrm{H} 22$ tumor-bearing mice $12 \mathrm{~h}$ after injection of CD44 and GA receptor-targeted nanoparticles (top) and free dye (bottom) (33). (C) Ultrasound-active nanobubbles targeted to FR and $\alpha_{v} \beta_{3}$ (right) accumulated to higher level in tumors than their nontargeted counterparts (left) (34). 
or even any given lesion, the biomarker expression of a cancer can vary widely. Thus, dual-targeted imaging agents may enable enhanced visualization by providing a larger number of potential binding sites. Additionally, they may find wider application across more patients due to their multiple specificities.

Although the use of two targeting ligands enables clearer visualization of cancerous lesions, this dual-targeting strategy also removes some of the in-depth information that is available from the use of a singly targeted agent. The use of radio- or fluorescently labeled compounds as companion diagnostics is gaining traction for patient stratification and treatment decisions (37); however, using an agent that binds to two different targets will make identification of patients with a given marker difficult, since it is challenging to tease apart which antigen is being recognized by the probe. Therefore, these dual-targeted imaging agents are likely to find success in localizing cancerous lesions, but not perhaps in selecting appropriate treatments for those patients, unless the treatment also uses a bispecific agent. They may, however, be useful for monitoring treatment response, to more readily visualize tumor burden over time.

An imaging tracer with high accumulation in a targeted site could additionally be modified for theranostic applications. This is becoming a common strategy with singly targeted entities - antibodies radiolabeled with imaging nuclides can be exchanged for therapeutic ones (38), additional therapeutic moieties can be attached (18), or the inherent theranostic capabilities of nanoparticles can be optimized $(13,27,29,30,33,34)$. Although a few preclinical therapeutic studies using dual-targeted platforms have been reported to date, this area is certainly waiting for exploration. Targeting of albumin in the bloodstream in addition to a cancer antigen has also been demonstrated to enhance the circulation of agents, enabling increased tumor exposure and uptake of therapeutic agents as well (39).

The bright future of dual-targeting strategies lies in their immense potential for improving imaging quality. A large number of dual-targeted strategies have already been reported in the recent literature, with more added each year. Enabled by this better tumor-to-background contrast, physicians will be able to more clearly visualize a patient's disease state and make more informed treatment decisions, making great strides toward personalized treatments. This high tumor-to-background should be exploited also for enhanced targeted therapies, whether that is through drug delivery, targeted radiotherapy, or other strategies. We expect that the successful translation of these agents into the clinic will enable earlier, more in-depth, and higher quality noninvasive imaging for cancer patients, thereby greatly improving the care they receive.

\section{DISCLOSURE}

This work was supported, in part, by the University of Wisconsin-Madison, the University of Pittsburgh, the National Institutes of Health (NIBIB/NCI P30CA014520,
T32GM008505, T32CA009206, R21EB020737, R21EB017317), National Natural Science Foundation of China (No. 81630049), and the American Cancer Society (125246-RSG-13-099-01CCE, ACS-RSG-17-004-01-CCE). No other potential conflict of interest relevant to this article was reported.

\section{REFERENCES}

1. Fan G, Wang Z, Hao M, Li J. Bispecific antibodies and their applications. J Hematol Oncol. 2015;8:130.

2. Mullard A. FDA approves first bispecific. Nat Rev Drug Discov. 2015;14:7.

3. Cole P. Duligotuzumab. Human anti-EGFR/anti-HER3 MAb, colorectal cancer therapy, head and neck cancer therapy. Drugs Future. 2015;40:167.

4. Yang Q, Parker CL, McCallen JD, Lai SK. Addressing challenges of heterogeneous tumor treatment through bispecific protein-mediated pretargeted drug delivery. J Control Release. 2015;220:715-726.

5. Huehls AM, Coupet TA, Sentman CL. Bispecific T-cell engagers for cancer immunotherapy. Immunol Cell Biol. 2015;93:290-296.

6. Yan Y, Chen X. Peptide heterodimers for molecular imaging. Amino Acids. 2011;41:1081-1092.

7. Eder M, Schafer M, Bauder-Wust U, Haberkorn U, Eisenhut M, Kopka K. Preclinical evaluation of a bispecific low-molecular heterodimer targeting both PSMA and GRPR for improved PET imaging and therapy of prostate cancer. Prostate. 2014;74:659-668.

8. Gai Y, Xiang G, Ma X, et al. Universal molecular scaffold for facile construction of multivalent and multimodal imaging probes. Bioconjug Chem. 2016;27:515-520.

9. Tateishi U, Oka T, Inoue T. Radiolabeled RGD peptides as integrin $\alpha_{\mathrm{v}} \beta_{3}$-targeted PET tracers. Curr Med Chem. 2012;19:3301-3309.

10. Yu Z, Ananias HJ, Carlucci G, et al. An update of radiolabeled bombesin analogs for gastrin-releasing peptide receptor targeting. Curr Pharm Des. 2013;19:3329_ 3341 .

11. Jackson $\mathrm{AB}$, Nanda PK, Rold TL, et al. ${ }^{64} \mathrm{Cu}-\mathrm{NO} 2 \mathrm{~A}-\mathrm{RGD}-\mathrm{Glu}-6-\mathrm{Ahx}-\mathrm{BBN}(7-$ 14) $\mathrm{NH}(2)$ : a heterodimeric targeting vector for positron emission tomography imaging of prostate cancer. Nucl Med Biol. 2012;39:377-387.

12. Li ZB, Wu Z, Chen K, Ryu EK, Chen X. ${ }^{18} \mathrm{~F}$-labeled BBN-RGD heterodimer for prostate cancer imaging. J Nucl Med. 2008;49:453-461.

13. Zhang J, Niu G, Lang L, et al. Clinical translation of a dual integrin $\alpha_{\mathrm{v}} \beta_{3}$ - and gastrin-releasing peptide receptor-targeting PET radiotracer, ${ }^{68} \mathrm{Ga}-\mathrm{BBN}-\mathrm{RGD}$. J Nucl Med. 2017;58:228-234.

14. Yu HM, Chen JH, Lin KL, Lin WJ. Synthesis of ${ }^{68} \mathrm{Ga}$-labeled NOTA-RGDGE11 heterodimeric peptide for dual integrin and epidermal growth factor receptor-targeted tumor imaging. J Labelled Comp Radiopharm. 2015;58: 299-303.

15. Luo H, Hong H, Yang SP, Cai W. Design and applications of bispecific heterodimers: molecular imaging and beyond. Mol Pharm. 2014;11:1750-1761.

16. Kwon LY, Scollard DA, Reilly RM. ${ }^{64} \mathrm{Cu}$-labeled trastuzumab fab-PEG24-EGF radioimmunoconjugates bispecific for HER2 and EGFR: pharmacokinetics, biodistribution, and tumor imaging by PET in comparison to monospecific agents. Mol Pharm. 2017;14:492-501.

17. Razumienko E, Dryden L, Scollard D, Reilly RM. MicroSPECT/CT imaging of co-expressed HER2 and EGFR on subcutaneous human tumor xenografts in athymic mice using ${ }^{111} \mathrm{In}$-labeled bispecific radioimmunoconjugates. Breast Cancer Res Treat. 2013;138:709-718.

18. Ding L, Tian C, Feng S, et al. Small sized EGFR1 and HER2 specific bifunctional antibody for targeted cancer therapy. Theranostics. 2015;5:378-398.

19. Razumienko EJ, Scollard DA, Reilly RM. Small-animal SPECT/CT of HER2 and HER3 expression in tumor xenografts in athymic mice using trastuzumab Fab-heregulin bispecific radioimmunoconjugates. J Nucl Med. 2012;53:19431950.

20. Luo H, England CG, Goel S, et al. ImmunoPET and near-infrared fluorescence imaging of pancreatic cancer with a dual-labeled bispecific antibody fragment. Mol Pharm. 2017;14:1646-1655.

21. Luo H, Hernandez R, Hong H, et al. Noninvasive brain cancer imaging with a bispecific antibody fragment, generated via click chemistry. Proc Natl Acad Sci USA. 2015;112:12806-12811.

22. Xin X, Sha H, Shen J, Zhang B, Zhu B, Liu B. Coupling GdDTPA with a bispecific, recombinant protein antiEGFRiRGD complex improves tumor targeting in MRI. Oncol Rep. 2016;35:3227-3235.

23. Lehmann S, Perera R, Grimm HP, et al. In vivo fluorescence imaging of the activity of CEA TCB, a novel T-cell bispecific antibody, reveals highly specific tumor targeting and fast induction of T-cell-mediated tumor killing. Clin Cancer Res. 2016;22:4417-4427. 
24. Warnders FJ, Waaijer SJ, Pool M, et al. Biodistribution and PET imaging of labeled bispecific T cell-engaging antibody targeting EpCAM. J Nucl Med. 2016;57:812-817.

25. McCormack E, Adams KJ, Hassan NJ, et al. Bi-specific TCR-anti CD3 redirected T-cell targeting of NY-ESO-1- and LAGE-1-positive tumors. Cancer Immunol Immunother. 2013;62:773-785.

26. Conde J, Dias JT, Grazu V, Moros M, Baptista PV, de la Fuente JM. Revisiting 30 years of biofunctionalization and surface chemistry of inorganic nanoparticles for nanomedicine. Front Chem. 2014;2:48.

27. Li L, Song L, Yang X, et al. Multifunctional "core-shell" nanoparticles-based gene delivery for treatment of aggressive melanoma. Biomaterials. 2016;111:124-137.

28. Shi S, Zhou M, Li X, et al. Synergistic active targeting of dually integrin alphavbeta3/CD44-targeted nanoparticles to B16F10 tumors located at different sites of mouse bodies. J Control Release. 2016;235:1-13.

29. Shu C, Sabi-Mouka EMB, Wang X, Ding L. Self-assembly hydrogels as multifunctional drug delivery of paclitaxel for synergistic tumour-targeting and biocompatibility in vitro and in vivo. J Pharm Pharmacol. 2017;69:967-977.

30. Lee JY, Termsarasab U, Park JH, et al. Dual CD44 and folate receptor-targeted nanoparticles for cancer diagnosis and anticancer drug delivery. $J$ Control Release. 2016;236:38-46.

31. Zhao HX, Yang CX, Yan XP. Fabrication and bioconjugation of BIII and CrIII co-doped $\mathrm{ZnGa} 2 \mathrm{O} 4$ persistent luminescent nanoparticles for dual-targeted cancer bioimaging. Nanoscale. 2016;8:18987-18994.
32. Zhang MZ, Li C, Fang BY, et al. High transfection efficiency of quantum dotantisense oligonucleotide nanoparticles in cancer cells through dual-receptor synergistic targeting. Nanotechnology. 2014;25:255102.

33. Mezghrani O, Tang Y, Ke X, et al. Hepatocellular carcinoma dually-targeted nanoparticles for reduction triggered intracellular delivery of doxorubicin. Int J Pharm. 2015;478:553-568.

34. Luo W, Wen G, Yang L, et al. Dual-targeted and pH-sensitive doxorubicin prodrug-microbubble complex with ultrasound for tumor treatment. Theranostics. 2017;7:452-465.

35. Rangger C, Helbok A, Sosabowski J, et al. Tumor targeting and imaging with dualpeptide conjugated multifunctional liposomal nanoparticles. Int J Nanomedicine. 2013;8:4659-4671.

36. Doolittle E, Peiris PM, Doron G, et al. Spatiotemporal targeting of a dual-ligand nanoparticle to cancer metastasis. ACS Nano. 2015;9:8012-8021.

37. Puranik AD, Kulkarni HR, Baum RP. Companion diagnostics and molecular imaging. Cancer J. 2015;21:213-217.

38. Razumienko EJ, Chen JC, Cai Z, Chan C, Reilly RM. Dual-receptor-targeted radioimmunotherapy of human breast cancer xenografts in athymic mice coexpressing HER2 and EGFR using ${ }^{177} \mathrm{Lu}$ - or ${ }^{111}$ In-labeled bispecific radioimmunoconjugates. $J$ Nucl Med. 2016;57:444-452.

39. Kelly JM, Amor-Coarasa A, Nikolopoulou A, et al. Dual-target binding ligands with modulated pharmacokinetics for endoradiotherapy of prostate cancer. J Nucl Med. 2017;58:1442-1449. 